\title{
Mobile game-based learning with local content and appealing characters
}

\section{Norshuhada Shiratuddin* and Syamsul Bahrin Zaibon}

Building of Information Technology, College of Arts and Sciences, Universiti Utara Malaysia,

Sintok, Kedah, Malaysia

E-mail: shuhada@uum.edu.my

E-mail: syamsulbahrin@uum.edu.my

*Corresponding author

\begin{abstract}
In tapping the thriving mobile business, a study on mobile content development, specifically local ones is urgently required. Highly wanted local contents development is indeed for education, entertainment and games. Thus, we develop a mobile game-based learning (mGBL) about local content, called MaCMoG. It was technically tested for its functionality, compliance, compatibility and soaking. Furthermore, to assess the insights of potential players, informal interviews and observations were conducted. In addition to that, the local game characters and the game plan developed for the mGame were tested for their appealing merit. Overall, responses were remarkably consistent. Participants overwhelmingly considered $\mathrm{MaCMoG}$ as interesting and really attractive, successful in delivering the intended learning content, easy to play and simple to use. Findings also stressed two important points (1) local, appealing and well-developed characters are important elements to ensure better learning experience and (2) local uniqueness must always be incorporated to ensure better learning motivation and increase mGBL acceptance.
\end{abstract}

Keywords: mContent; mobile content; mGBL; mobile game-based learning; digital content.

Reference to this paper should be made as follows: Norshuhada, S. and Zaibon, S.B. (2010) 'Mobile game-based learning with local content and appealing characters', Int. J. Mobile Learning and Organisation, Vol. 4, No. 1, pp.55-82.

Biographical notes: Norshuhada Shiratuddin is an Associate Professor and Applied Science Chair at the Graduate Department of Computer Science, College of Arts and Sciences, Universiti Utara Malaysia. She has published more than 100 papers in eContent engineering, mobile and web application design, e-learning and HCI.

Syamsul Bahrin Zaibon is a Lecturer at the Graduate Department of Computer Science, College of Arts and Sciences, Universiti Utara Malaysia. Currently, he is pursuing his $\mathrm{PhD}$ programme in Information Technology. His current research focuses on educational mobile game.

Copyright (c) 2010 Inderscience Enterprises Ltd. 


\section{Introduction}

To date, there has been no universal definition of 'electronic or digital content'. However, it is acceptable to define 'content' as:

"any sound, text, still picture, moving picture or other audio-visual representation, tactile representation or any combination of the preceding which is capable of being created, manipulated, stored, retrieved and communicated electronically" (Malaysia Communications and Multimedia Act, 1998)

The examples of electronic content are broadcasting, film, video, visual arts, digital TV, online games, digital content publishing, broadband content development, online education content, digital creative media and industry-related software application (Md Saidin and Amira, 2006; Roslan, 2007). Recently, the market size of content is increasing exponentially, and these are achieved through film, TV content, business content, education, training and games (Jo, 2007; W2Forum.com, 2005). However, for mobile content (mContent), which is defined as any type of media viewed or used on mobile phones, the most popular downloads are ring tones, wallpapers, music, gambling and games (Jo, 2007). Picking up pace are news and sports (Budde, 2007).

The ownership of mobile telephony in Malaysia is growing at a much faster rate than the internet subscription. This is due to the fact that mobile nature of hand telephony and the ease of communicating have made it almost a necessity. The mobile sector grew from over 2 million subscribers in 1998 to 15 million, a penetration of $60 \%$, by early 2005 (Budde, 2005). Now, in 2007 the penetration has grown to about 84\% (Budde, 2007). Malaysia is the second highest mobile penetration in South East Asia after Singapore.

Hence, in tapping the thriving mobile business, a study on mContent development, specifically local mContent is urgently required. The Malaysian government has come up with a strategy called MyICMS 886, which dictates a number of strategies for the growth of local content and these are promoting awareness, building competencies in higher education and forming strategic alliances. The goals are to encourage local creativity, address new content demands and also export content. In achieving these goals, the Malaysian government together with a number of key industry players have provided many funding incentives specifically for local content production. This is expected to provide adequate local contents that reflect Malaysian culture and values. It is also expected that the contents should be sold as additional services by mobile operator to broaden phone usage (Pelkonen, 2004).

In our local scene, Roslan (2007) reports that a highly wanted local content development is indeed for education, entertainment and games. Thus, the main aim of this study is to develop an interactive, animated and informative mobile game (mGame) about local content that is appealing to the children as a learning tool. The target group of our study was children between 9 and 12 years old.

This paper is organised as follows. Section 2 elaborates the many issues associated with mContent and mGame. In particular, the history, technologies, characteristics and trends of mGame are presented. Section 3 describes mobile games in learning environment. Then, Section 4 informs readers about the methods and activities involved in accomplishing all research objectives. mGame that was produced is discussed in Section 5. Section 6 outlines the summary of the outcomes of this study. 


\section{Review of mContent and mGame}

mContent is defined as any type of media which is viewed or used on mobile phones. The examples of the few mContent are ring tones, graphics, games, movies, news, sport scores and music used or stored in mobile devices. Mobile music is normally formatted as an advanced audio coding (AAC) file or an MP3, and comes in several different formats. Mobile video comes in several forms including 3GPP, MPEG-4, RTSP and Flash Lite. Also, mContent can be categorised into the following (Yuan, 2003):

1 Infotainment: sports, city guides, news, weather, directory, maps, traffic alerts, cinema, theatre, clubs, museums, videos, cartoons, graphics, icons, jokes and dating.

2 Multimedia: audio and video streaming, animations.

3 Gaming: games and gambling services.

4 Location-based services (LBS), mCommerce and other services: LBS, local information, travel, interactive maps, vehicle positioning, banking and brokerage.

5 mCommerce: promotions, shopping, price comparisons, advertising/sponsoring and ticketing.

Usually, these contents are downloaded through WAP sites, however recently users can browse a huge database for mContent from Telcos (such as Maxis and Digi in Malaysia) or phone companies (e.g. Nokia and Samsung) and directly download their choices to their handsets. Among the above-described categories, this study identified mGame as the focus of research, thus next section presents the reviews of some aspects of the technology.

\section{1 mame}

The typical definition of mGame is games developed to run on mobile or 'cellular' phones (Mencher, 2004). Similarly, IBM defines mGame as games developed or adapted specifically for phones or other mobile devices. Also, mGames can be applications that allow people to play a game on a mobile device. mGames can be classified into three broad categories (Chauhan, 2007). The first is embedded games, that is, games that are embedded into the mobile device's system: a good example is Snake, shipped with all Nokia phones. The second is the SMS games, often in the form of live contests and polls. The last is browser games, played using mobile phone's built-in microbrowser, either online or offline mode. In addition to the above classifications, mGame genres can be divided as follows:

1 arcade/action - for example Doom and Alien

2 sports - bowling, golf and football

3 skill, strategy and logic - for instance Sudoku

4 card and board - like monopoly

5 role playing game - for example Final. 
mGame is also associated with 'power' or 'casual' games. Casual are non-violent games that are simple to learn, however difficult to master. These are categorised as games that players can leave and pick up again easily. Play time can vary from a few minutes to hours. Power is the opposite of casual.

mGames may be played using the communications technologies present in the device itself, such as by text message (SMS), multimedia message (MMS) or GPRS location identification. More common, however, are games that are downloaded to the mobile phone and played using a set of game technologies on the device. The mGames are usually downloaded via the mobile operator's radio network, and also loaded into the mobile phones when purchased, or via infrared connection, Bluetooth or memory card.

Informa Telecoms and Media once stated that the worldwide market for mGames is predicted to grow from US\$2.41 billion in 2006 to US\$7.22 billion by 2011. Another well-known research company, Juniper Research, also projected the global revenues of mGame will grow from US\$3 billion in 2006 to US $\$ 17.5$ billion by 2010 . On the same issue, Asia Pacific region, especially Japan and South Korea will dominate the mame market. Asia also is said to contribute $38 \%$ of global revenues over the whole forecast period.

This exceptional growth is due to a number of driving factors, namely,

1 anytime, anywhere entertainment

2 hybrid devices

3 enhanced infrastructure capabilities.

Growth area is also expected to come from several mobile groups of gamers, depending on their addiction to gaming (Semenov, 2005): casual, youth and hardcore gamers, with the last one dominating the mass market. Moreover, phones are portable, networked and more than one billion mobile phones are in use today (Mencher, 2004).

\subsection{History of mGame}

Mobile gaming history starts back in 1980s when Nintendo introduced its portable LCD game series 'Games and Watch', where characters like Mario was introduced (Pelkonen, 2004). Then later Nintendo introduced a new kind of game console: Gameboy-console with changeable cartridges. However, in 1997 Nokia developed a new kind of game that could be played by using a mobile phone. The first game was called Snake and although it was very simple it became surprisingly popular. Since then Nokia developed more similar games for their phones, which are either permanently installed on the phones or downloadable.

Late 1990s, new types of mobile gaming emerged, which allow gamers of multiplayer gaming. These games were WAP-games and SMS-based games. Next, in 2001, when mobile phones became equipped with colour screens, Java games were introduced to the market. When more advanced phones (such as smart phones) were produced capable of supporting 3D-graphics and natural sounds, Nokia introduced N-Gage platform and wireless online gaming with N-Gage Arena (Pelkonen, 2004). Currently, mobile gamers have the opportunity to sample much more innovative choices of mGames, such as location-based games (Bell et al., 2006). 


\section{3 mame platforms}

Platform acts as games console inside mobile phones. Though the mGame surroundings is undoubtedly changing fast, it is fairly safe to narrow down the platforms to Windows Mobile, Sun's J2ME (Java 2 Micro Edition, or 'Java ME'), Macromedia's Flash Lite, Palm OS, Symbian OS, DoCoMo's DoJa, Qualcomm's BREW (Binary Runtime Environment for Wireless), Wireless Internet Platform for Interoperability (WIPI) and Execution Environment (ExEn) (a proprietary piece of software developed by games publisher In-Fusio). WIPI is a middleware platform used in South Korea that allows mobile phones, regardless of manufacturer or carrier, to run internet applications. Other platforms such as Android (a new Linux-based platform), Mophun and .NET Compact Framework are also available, but not as common. The different types of platform mean they have distinct capabilities and their games are not interchangeable.

Briefly, BREW is a platform that allows for several different languages to work with a mobile device, including $\mathrm{C}++$, Java, XML, Flash, and so on. However, BREW is difficult to learn. Similarly, Symbian is difficult to learn and also it has poor debugger support. The dominant mobile software platform is J2ME and it has a set of specifications that allow for a subset of the normal Java classes to work with a particular group of devices (Abolins, 2006). So, this allows a short learning time.

Flash environment is ideal for 2D graphics heavy options with a market that can support the Flash Lite player. In addition to providing many widgets, it has extensive developer community and support. Any mGame developed for this platform, the market penetration is on the average side. It is also easy to learn as it is ActionScript based.

All the above-mentioned platforms are supported in most mobile phones, and they all provide free tools and documentation for developers as well.

\section{4 mame content creation}

Creating content for mGame requires specific skills and knowledge, which normally include the technical knowledge in designing and creating graphics, animations, audio, sound effects and the level of interactivity to bring out the excitements and fun. Also, literatures suggest that constraints such as screen resolutions, colours and sound, file size and latency, found in mobile devices have to be taken into account. Furthermore, storyline and gaming styles are also issues to be considered.

Developing storyline can be based on personal experience, ideas, famous movie shows, historical events or sports, and illustrated through a storyboard. Essentials elements to observe are that games are meant to be challenging, fun and entertaining for players.

Gaming styles involve

1 playing modes (i.e. single player, multiple players or location-based concept)

2 game setting

3 game structure.

While game setting concerns the graphics creations and the background design to provide appropriate gaming mood, game structure shows the flow and choices of game play. Multiplayer games are gaining interest, to take advantage of the ability to play against other people (Korhonen and Koivisto, 2007). 


\section{5 mame characteristics}

The tempting thing about mGames is their wide reach as mobile phone has become among the most commonly carried personal items. Unlike traditional console games that target geeks and teenagers, mGames are accessible to anyone (Yuan, 2003). mGames also present a big paradigm shift from console games due to the hugely different target audience, lifestyle and distribution models. According to Yuan, a successful mGame should be designed to have a number of characteristics including

1 easy to learn, which can be translated into having non-steep learning curves

2 providing rich social interactions to cater for the growing multiplayer games

3 interruptible so that children, teenagers or adults, who often have small chunks of free time can switch smoothly between tasks

4 take advantage of mobile device hardware and network protocols such as global positioning system (GPS) extensions and short message service (SMS)/multimedia message service (MMS) messaging

5 avoid explicit violent or sexual content.

Furthermore, mGames are often played at odd moments, for example, on bed, while waiting, travelling on a train, or even during 'killing time'. Therefore, literatures suggest that mobile games should be:

1 quick and easy to start, play and navigate (Forum Nokia, 2004)

2 easy to understand, where players should quickly grasp the procedures, concepts and processes used in playing (Desurvire et al., 2004)

3 compelling and challenging, but not impossible to win (Korhonen and Koivisto, 2007).

\section{6 m Game trend and habit}

Since mobile business is thriving, many mobile device and telecommunication companies have conducted research on mGame trend and habit, simply trying to look for users' attitudes and needs. Among these, the Nokia Corporation, who in 2006 reported the following findings from interviewing 1,800 respondents across six countries which are China, Germany, India, Spain, Thailand and USA:

- The mobile phone gamers surveyed are frequently playing mGames with the vast majority ( $80 \%$ ) playing at least once a week and $34 \%$ playing every day.

- The average length of time spent playing mGames is $28 \mathrm{~min}$.

- Nearly two-thirds (63\%) of the respondents preferred the richer experience of Nokia's next generation mGames offering over existing Java 2D and Java 3D games offerings, particularly following game trials.

- The majority (65\%) would prefer to pay for a full game outright rather than buy a subscription $(27 \%)$. 
- When it comes to getting games, over-the-internet (OTI) distribution (34\%) is almost as popular as over-the-air (OTA) distribution (45\%).

- Multiplayer gaming is a regular part of mobile phone gamers lifestyles with $45 \%$ playing multiplayer games on their mobile phones at least once a month.

- $62 \%$ would send demos of games they like directly to their friends and $79 \%$ would try a demo received from a friend.

Back in 2002, In-Fusio, a mobile games service provider, released a survey findings, whereby they found that majority of mobile players are adults under 34 , and nearly half of those are women (44\%). Their findings also revealed that mGames influence purchasing decision of devices and they are indeed viral.

Another study by RealNetworks ${ }^{\circledR}$, Inc. (2006), a publisher and distributor of casual games, stated that their research findings demonstrate USA adults who play computer games play 'casual' games for a quick mental break. About $64 \%$ of the 1,302 respondents cited game play as a way to unwind and relax, while $53 \%$ play for stress relief. They also revealed new details about the casual games social phenomenon that more than $70 \%$ of people buying casual games from the company are women age 40 and older. Among those with children, $75 \%$ see educational benefits for their children who play mGames. In agreement with the last sentence in the above paragraph, the focus of this study has been singled out to be mGame-based learning, which is also the growth area indicated in the Malaysia MyICMS 886.

\section{3 mGame-based learning}

A growing body of research indicates that mobile technologies can be effective tools in catering for students in a digital age and there are signs of the motivating potential and possible learning gains of games played on mobile devices with young adults (Cogoi et al., 2006; Fabricatore, 2000; Massie, 2005). Albernathy (2001) pointed out that if applied to educational settings, mGame has the potential to enrich the learning experiences of learners. Other several advantages of using mGames as an auxiliary to learning can be found in Lee (2005). This kind of learning is known as mobile game-based learning (mGBL) as pointed out by Cogoi et al. (2006).

mGBL is e-learning games using through mobile devices and wireless transmission (Lee, 2005; Sánchez et al., 2007), and it can be directed at children aged from 8 to 15, or young adults from 16 to 24 years or even older generations. mGames developed are mostly adaptations of popular computer games such as shooting games with guns and fighting games with other weapons. Mostly, they are simple entertainment-oriented games with added violence. Since, it is predicted that games will be a key element in the growth process of teenagers (Chen and Kinshuk, 2005) and game-based learning is an important alternative or supplement to traditional teaching (Antonellis et al., 2005), mGame-based learning should be further explored. Next section describes a few examples of such educational mGames. 


\subsection{Examples of $m G B L$}

There are a variety of mobile games that are being implemented to help educate young adults and help them to improve their literacy skills. For example, a quiz game that provides the user with a new mobile way of learning on how to drive a car, by testing theory-based question and text messaging quizzes, a mini language course (Attewell, 2005). However, this process of mobile learning is least popular because the cost of gaming increases with each SMS sent to the game server (Schwabe and Goth, 2005).

Emergency 112, an mGame to teach first aid techniques for medical emergencies created by the Spanish developer Exelweiss is also a good case in point (Bogost, 2006). The game was created for emergency response company Area de Intervención, but it is also generally available for the public. The player takes the role of a Good Samaritan who finds pedestrians in trouble. The player then must check the victim's condition and choose an appropriate diagnosis and treatment.

Next project that is rather interesting is by the ELIOS group at the University of Genoa, who has introduced a noteworthy innovation in mobile gaming technologies, called Venice Game (VeGame). The game offers a kind of 'electronic treasure hunt' through the narrow streets of Venice. As visitors make their way along a designated $3 \mathrm{~km}$ track, they are presented with a series of quizzes and riddles on Venetian art and history. The route it proposes is off the busy tourist track, bringing visitors into contact with lesser known, though nonetheless fascinating, treasures of this world-renowned city (Bellotti et al., 2003).

Language learning can benefit from mGame too. Kam et al. (2007) believes that mGames on cell phones have the potential to make English as a second language (ESL) learning resources more accessible to children in out-of-school settings. In particular, it appears to be a promising approach for complementing the existing formal schooling system. He developed a game where the player's avatar in the game is the local entrepreneur. He is required to travel to Kanpur by train to meet a business associate. The latter will be at the destination train station at $4 \mathrm{pm}$ on 24 September 2006 to wait for his arrival. The avatar must not be late, so as not to create a poor impression. The game starts with a scene of the train station at the originating city, where the avatar is required to purchase a train ticket to Kanpur. Suppose that he is at the ticket counter engaging in a dialogue with the clerk non-player character (NPC) in English, such that multiple-choice options are provided for possible responses that he can select via the keypad.

History also can take advantage of mGame as shown by Ardito et al. (2007). They designed an mGame to support young students learning history at an archaeological site, by exploiting mobile technology. The approach uses game-play, since it stimulates in young students an understanding of history that would otherwise be difficult to engender, helping players to acquire historical notions and making archaeological visits more effective and exciting. Strength of the system is that, by running on the visitors own cellular phones; it requires minimal investments and small changes to the existing site exhibition.

Another good example is described by Schwabe and Goth (2005) called 'MobileGame'. It is a prototype of an orientation game in a university setting. Schwabe and Goth developed scenario and the general architecture of the prototype, focusing also on the evaluation of design issues and the effects observed in two trials. Their study revealed a number of design issues including supporting work on the move poses difficult interface questions, the accuracy of current outdoor and indoor positioning systems is still 
problematic and the game requires near real-time response time. The evaluation of the effects shows that features such as 'map-navigation' and 'hunting and hiding' lead to excitement and fun. The game success is based on the motivating design of the game itself.

Other area that could utilise mGame is culture study. Maniar and Bennett (2007) propose an educational mGame called C-Shock that reduces culture shock by teaching the user about the specific culture in question. The game is targeted at helping international students cope with 'culture shock' and university life in Britain. It includes moments of cultural awakening like going to the pub or watching people being affectionate to each other in public. This concept is based on the notion that not only can a computer game provide a safe place for exploring cultural differences (Raybourn and Wærn, 2004) but also games potentially provide an excellent learning environment because they motivate a person and hold their attention (Amory and Seagram, 2003; Prensky, 2001).

To conclude this section, a number of mGBL were studied. This furnishes us with ideas, features, functionality, game plan, storyline and content suitable for development. It also assists the decision-making process in identifying what is considered as among the appealing storylines for Malaysian local content industry. For the purpose of our study, we decided on developing mGBL for casual players.

\section{Game design and development}

mGame development is more difficult than desktop application development (Zhang et al., 2007). Its challenges are smaller screen size, low colour depth, limited memory, limited processor, limited file size, short battery life, few input style options and different fragmentation (Janousek, 2007). Due to these, literatures suggest for casual game, game plan should be simple and addictive over graphics game, and also uses simple user controls (Dholkawala, 2005; Koller, 2003; Novak, 2004). Furthermore, the file size should be aimed for less than $200 \mathrm{~kb}$.

Janousek (2007) compared two most popular platforms used for mGame development, J2ME and Flash Lite. He concluded that Flash platform offers one of the best environments for rapid mGame development for casual type content based on the factors depicted in Figure 1.

Figure 1 A comparison between J2ME and Flash

\begin{tabular}{|l|l|}
\hline \multicolumn{1}{|c|}{ Java } \\
J2ME \\
Device dependent \\
High fragmentation \\
Complex \\
Powerful \\
2D \& 3D \\
\hline
\end{tabular}


For the above reasons, the mGBL developed for this study opted for the Flash platform. Henceforth, this section explains the design and development phase which includes five main stages:

1 characters development

2 game conception

3 game plan and storyline design

4 integration of characters, game plan and storyline

5 porting and testing of game.

\subsection{Characters development}

The main cast was decided to be a male stick figure that portrays a Malay ethnic group. The character was named LidiMan which comes from two words 'Lidi' and 'Man'. 'Lidi' is a Malay word which means stick.

A stick figure is a very basic type of drawing which is $2 \mathrm{D}$ perspectives; generally all parts of the figure are represented by single straight lines. There are many interesting animations that have been done using this kind of animation such as Stick Page (http://www.stickpage.com), Stick Figure Ninja (http://www.stickfigureninja.com) and Stick Figure Productions (http://www.stickfigureproductions.com). LidiMan was designed in 2D perspective so that it can be broken down into smaller pieces, reused, recombined to form new elements, borrowed by other project and enhanced to other fields.

Character development is the stage where we design, characterise and construct the personality of the animation characters. The basic principles involved in the character development are the use of spheres, cylinders, basic perspective, anatomy and proportions. The LidiMan character was developed using the steps proposed by Ward (2005) and Kneunster (2004). These steps are:

1 research

2 preparation

3 design.

In the research step, information about the main character such as biography and personality traits is gained. From this step, the personality types of the main character as well as the others are identified for various character personalities.

In the preparation step, the information and sources of images related to the character are compiled. The rules of $2 \mathrm{D}$ perspective are used to produce solid designs. Besides, costumes are also created by employing a diverse range of techniques and processes to synchronise with the game concept. Identifying the appropriate costume designs for the character is based on cultural and historical reference. In our case, these are Malay, Chinese and Indian; the main ethnic groups in Malaysia.

In the design step, character is sketched, drawn and coloured until satisfied. Figure 2 illustrates the early sketches of the character development. The early sketches as suggested by Agustin et al. (2007) can give a clear illustration which is intended to provoke more discussion about the character development. These sketches can be then 
used as a starting point for further refinement or as a reference for the development. This is true because we can have the overview of different perspectives (body proportions) of that particular character.

Thus, when the final sketches are finished, using animation software, the characters are given 'life'. Figure 3 shows the detail steps involved in LidiMan character development with different facial expressions and body positions. Although, the sketches are designed nicely, the restriction on the file size for the game needs to be considered next at the production stage.

Figure 2 Early sketches

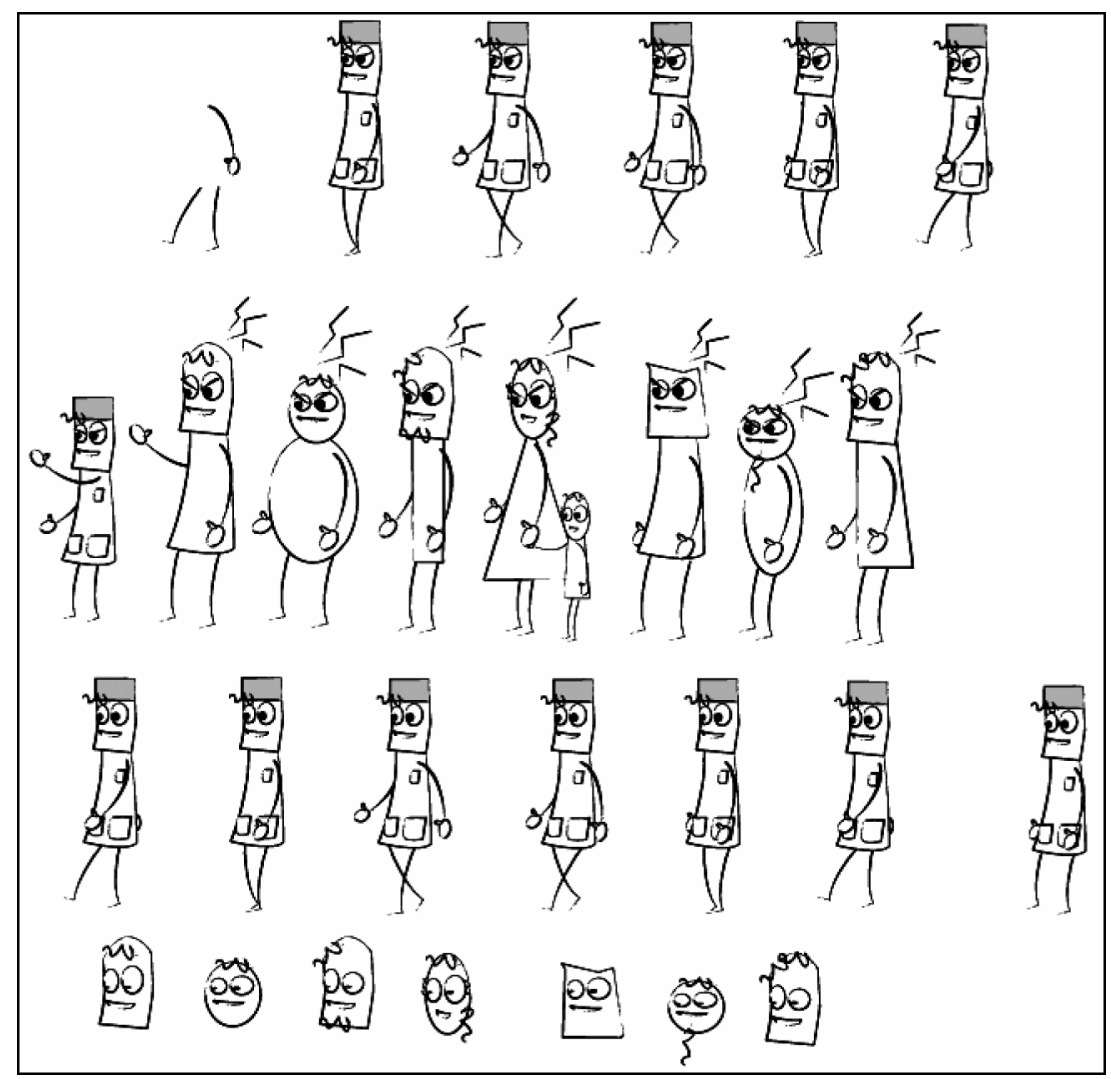

Figure 3 Facial expression development of LidiMan

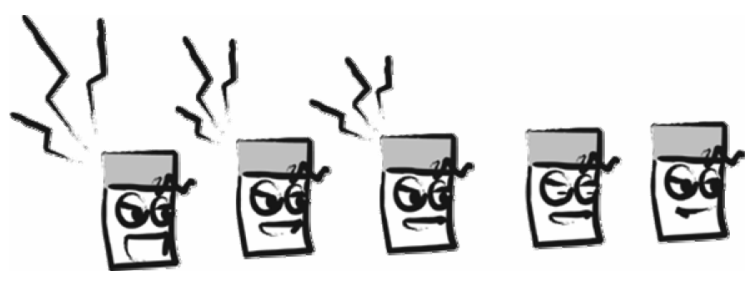


Once all characters have been designed, an approach to cast them to potential audience was planned. This is important to see whether the characters meet the requirements of appealing to the audience. So, during this stage, a field test was carried out for the purpose of determining whether characters designed meet the appealing dimensions. At this point, an animated electronic book (eBook) containing characters and similar storyline to the game was produced. It was shown to 43 primary school students, aged 9-12. This age group was selected as a representation of our research target group. A three-point scale instrument was developed and utilised. The dimensions of appealing are based on its definition as found in a number of well-recognised dictionaries. Figure 4 illustrates one example of the question in the instrument. The three-point scale was matched and represented by 'smiley faces' to better relate the scale to children (Ransley, 1991). Tables 1 and 2 indicate the findings of the appealing dimensions.

Figure 4 Instrument utilised to check appealing dimensions of game characters (see online version for colours)

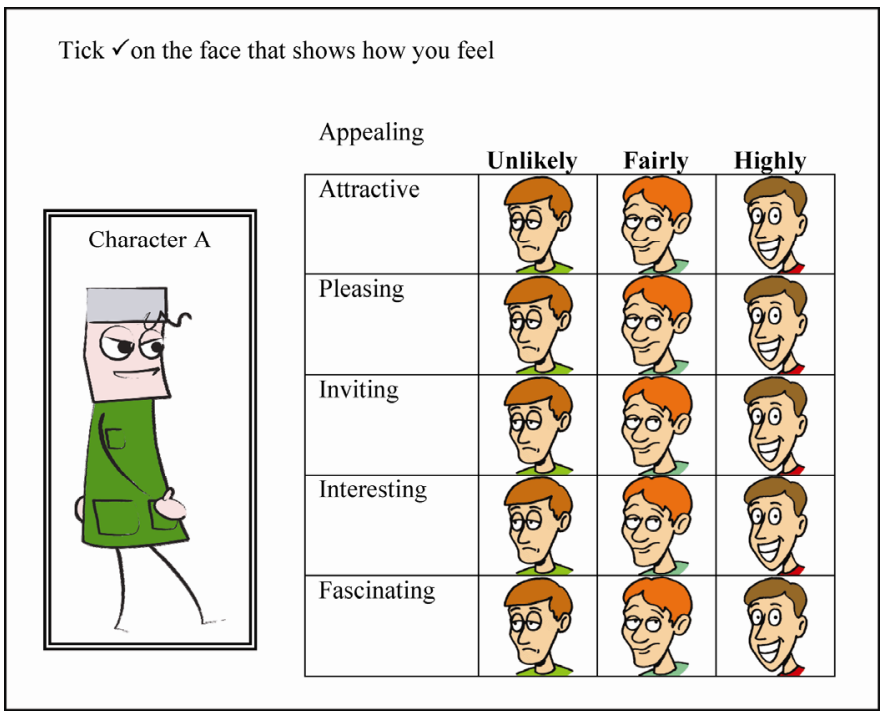

Table 1 Findings of school students participated in answering the appealing instrument

\begin{tabular}{lccccc}
\hline Age group & No. of students & Gender & No. of students & Race & No. of students \\
\hline 9 & 11 & Male & 22 & Malay & 22 \\
10 & 12 & & & Chinese & 10 \\
11 & 10 & Female & 21 & Indian & 9 \\
12 & 10 & & & Others & 2 \\
Total & 43 & Total & 43 & Total & 43 \\
\hline
\end{tabular}


$m G B L$ with local content and appealing characters

Table 2 Findings of appealing dimensions

\begin{tabular}{|c|c|c|c|c|c|c|c|c|c|c|c|c|c|c|c|}
\hline \multirow{2}{*}{$\begin{array}{l}\text { Appealing } \\
\text { dimensions } \\
\text { Character name }\end{array}$} & \multicolumn{3}{|c|}{ Attractive } & \multicolumn{3}{|c|}{ Pleasing } & \multicolumn{3}{|c|}{ Inviting } & \multicolumn{3}{|c|}{ Interesting } & \multicolumn{3}{|c|}{ Fascinating } \\
\hline & $U$ & $F$ & $H$ & $U$ & $F$ & $H$ & $U$ & $F$ & $H$ & $U$ & $F$ & $H$ & $U$ & $F$ & $H$ \\
\hline LidiMan & & & 43 & & & 43 & & & 43 & & & 43 & & & 43 \\
\hline Malay Dad & & & 43 & & & 43 & & & 43 & & & 43 & & & 43 \\
\hline Indian Dad & & & 43 & & 9 & 34 & & 7 & 36 & & 7 & 36 & & 11 & 32 \\
\hline Chinese Dad & & & 43 & & & 43 & & & 35 & & & 36 & & & 33 \\
\hline Chinese Mom & & 43 & & & 43 & & 14 & 29 & & 43 & & & 40 & 3 & \\
\hline Malay Granddad & & & 43 & & & 43 & & & 43 & & & 43 & & & 43 \\
\hline Malay Mom & & & 43 & & & 43 & & & 43 & & & 43 & & & 43 \\
\hline Malay Baby Girl & & & 43 & & 3 & 40 & & 3 & 40 & & 3 & 40 & & 3 & 40 \\
\hline Malay Baby Boy & & & 43 & & & 43 & & & 43 & & & 43 & & 3 & 40 \\
\hline
\end{tabular}

Note: U - Unlikely; F - Fairly; H - Highly Likely.

Figure 5 Development of other characters (see online version for colours)

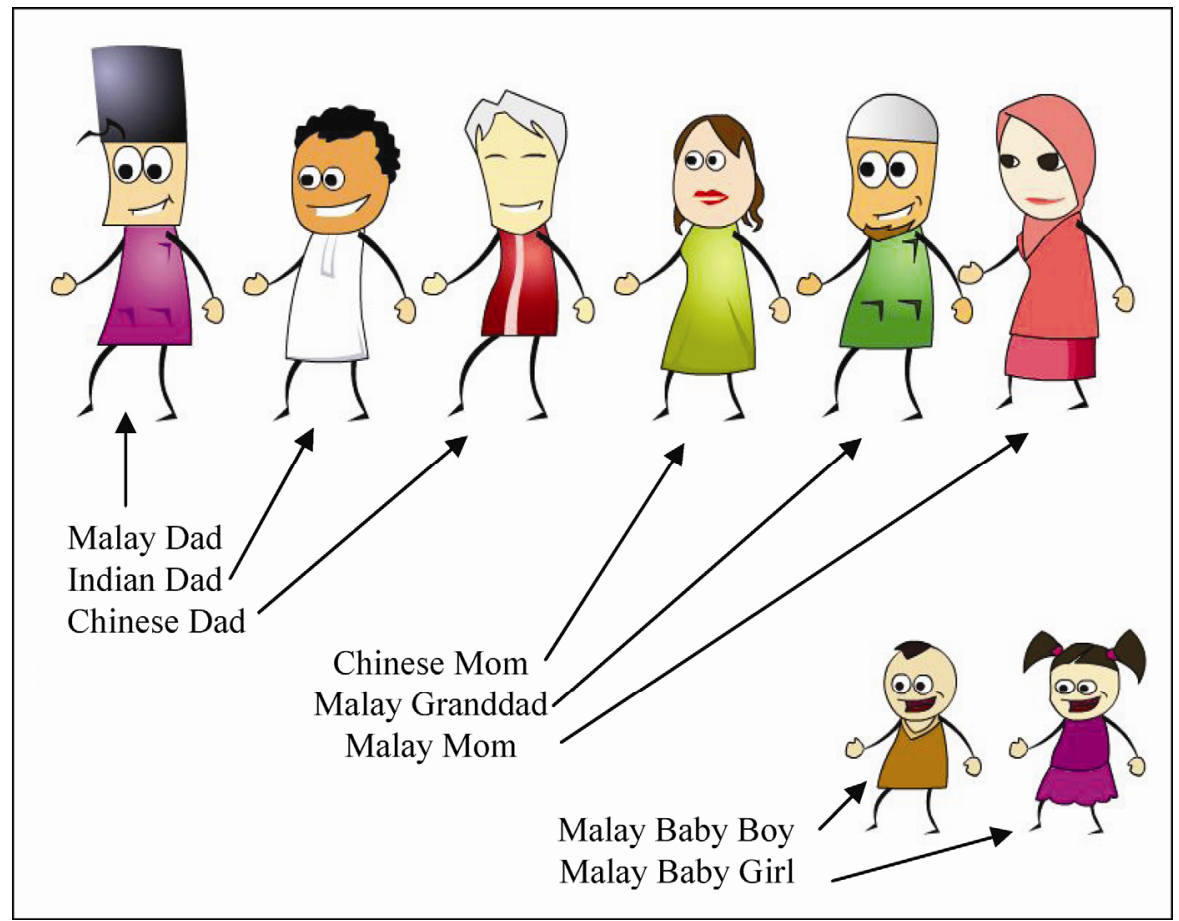

In addition to the main character, LidiMan, eight more characters were evaluated for their appealing dimensions. These characters are illustrated in Figure 5. Apart from these characters, additional objects such as mosque, cat, kite, trash, money, house, fruit and 
trees were also sketched. These sketches became instruments to collaboratively support the primary characters. For instance, these sketches can be used and transformed from one stage to other stage during the game development.

As it can be seen from Table 2, it can be concluded that a huge majority of the students see all characters, except the Chinese Mom, as highly appealing. The Chinese Mom was not considered highly appealing because the students did not see her reflecting as a Chinese woman. Although, this was the case, the Chinese Mom was still put into the game plan as a higher number of students considered her 'fairly appealing' when compared to the 'unlikely' results.

\subsection{Game conception}

The conception process is the very first phase of the game design practice. Here, deciding contents for the game involved making many choices concerning technical, aesthetic and usability concerns. Certainly, the focus is to distinguish the characteristics and qualities of the animation as a medium for disseminating information. Developing high-value content that advances educational strategy and tactics presents two key aspects (Di Blas et al., 2005). The first aspect is to determine the content that provides the greatest value. The second aspect is to determine in what manner to produce the content. Certainly, the highest-value content is that which directly impacts targeted audience (Di Blas et al., 2005), which in our case local content about Malaysian. Consequently, the content for the game was focused on instilling Malay culture and values.

Zapping generations (i.e. youngsters nowadays), with diversity of cultures and races, are less interested in learning about national cultures and customs. They are impatient, hungry for information and accustomed to digital media. They also have neither time nor enough patience to read and digest large amounts of information (Norshuhada et al., 2007). Information delivery should be made convenient, attractive, creative, easier to understand, entertaining, simple, suitable for different age groups, cheap to get or produce, humorous, effective and appealing.

Consequently, casual game that combines education with entertainment aspects and instils cultural information is found to be one of the main ways to promote and enhance the learning environment to be much more engaging and fun-filled. The game was designed to be fairly simple. In general, the game concept is called a 'side-scroller'. The player controls a single character, which is LidiMan. The primary objective is to behave positively while finding his way home and of course, the player must reach the end of each level.

\subsection{Game plan and storyline development}

After the characters have been developed and game concept decided, the next stage is the game plan and storyline development. In our study, the storyline is set on the main character (LidiMan), who is lost and thus he needs to find his way home. The aim of the game is to educate children on positive and negative social values. Along the way, he comes across people with different characters and situations. For each encounter, LidiMan is given options to make a decision for the appropriate action to be taken. 
A player is awarded with points whenever he chooses the right option and vice versa. The following is a sample of the game plan document produced for our mGame.

Game Concept:

Game Theme:

Educational Value:

Character Name:

Environment:

Storyline:
Side view 2D, Platform Game

Educational Game

Learn about Malay Culture

LidiMan, Male, Malay

At village views.

LEVEL 1

Lidiman is lost and thus he needs to find his way home. Along the way, he comes across people with different characters and situations. For each encounter, he is given options to make a decision for the appropriate action to be taken. He is awarded with points whenever he chooses the right option and vice versa.

\section{The Contents:}

Encountered characters that give points:

1. If LidiMan comes across an elderly person, he should...

a. Option 1: bow to show respect

b. Option 2: continue walking

2. If LidiMan encounters a Malay man/woman, he should...

a. Option 1: greet him/her with 'salam'

b. Option 2: say 'Hello'

3. If LidiMan encounters a cat on a tree, ...

a. Option 1: he should walk pass the tree

b. Option 2: he should help the cat

4. If LidiMan find some cash, ...

a. Option 1: he should pick it up

b. Option 2: he should leave it

5. When LidiMan encounters a Chinese many, he should...

a. Option 1: greet her with 'salam' and shakes hands

b. Option 2: greet her with 'salam' without shaking hands 
In short, our mGame is called MaCMoG (Malay Culture Mobile Game). The values that are incorporated in the game contents provide the basis of the game play rules and actions. The following are examples of such values incorporated in $\mathrm{MaCMoG}$ :

- $\quad$ Respecting the elderly: when walking in front of the elderly, one should bow.

- $\quad$ Caring of environment: one should pick up trash.

- Caring of animals: abandoned and in distress animals must be helped.

- Honesty and responsibility: one should not take other people's belonging.

- Self-discipline: self-discipline is important in the daily activities.

- Helping hands: always help people in need.

\subsection{Integration of characters, game plan and storyline}

In integrating the characters, game plan and storyline that have been created, first phase at this point, is to plan for the game architecture and its physics and logic game features.

The game architecture consists of main components or features of the game, system functionalities and the requirement of hardware and software. Its purpose is to maintain the consistency throughout the entire game design and development. Figure 6 shows the game architecture which was utilised in this project. It consists of character, movement style, scoring data, mobile device and user interface system. Each of the game components contribute to the successful game play from the user because the development of the game is solely referred to this architecture.

Figure 6 Game architecture

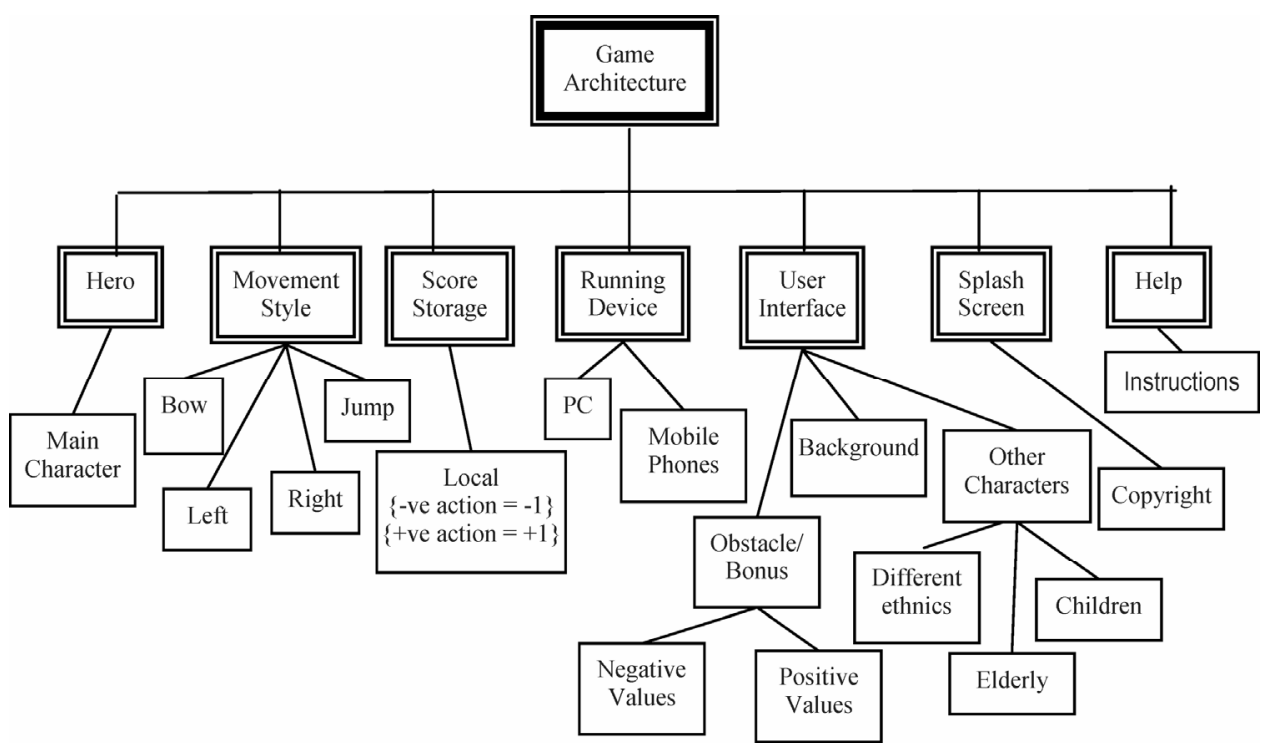


Physics and logic game features are important to game architecture. A physics game feature is a component that controls the physics variables such as gravity and jumping which can simulate effects for the game. It can be used to enhance the way it imitates the real world, to create pleasant dream world effects or to produce highly realistic games and animations.

In addition to the physics game features, the logic game feature also gives the game an ability to reach unprecedented levels of realism in logical rules of the real world such as moving forward or backward and incrementing score data. Both game physics and logic features can be scripted via Flash ActionScript for functionality during the coding part. For this reason and also the capabilities of Flash for making game development much simpler while supporting cross-platform development ability from PC to mobile, Adobe Flash Lite $^{\mathrm{TM}}$ was chosen as the game development tool.

The following screen shots (Figures 7-12) illustrate the user interface and screens of the mGame. At first, when the game is loaded, the main page is displayed. Players can continue to start playing the game by pressing start button or selecting other buttons for instructions. The game will start at first level, and players can control the game by pressing the arrow and selection keys.

Figure 7 Main page of $\mathrm{MaCMoG}$ (see online version for colours)

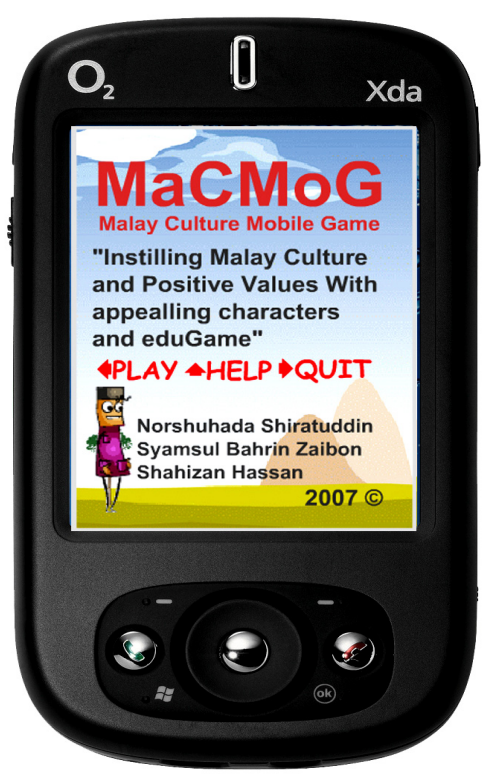


Figure 8 Instruction page (see online version for colours)

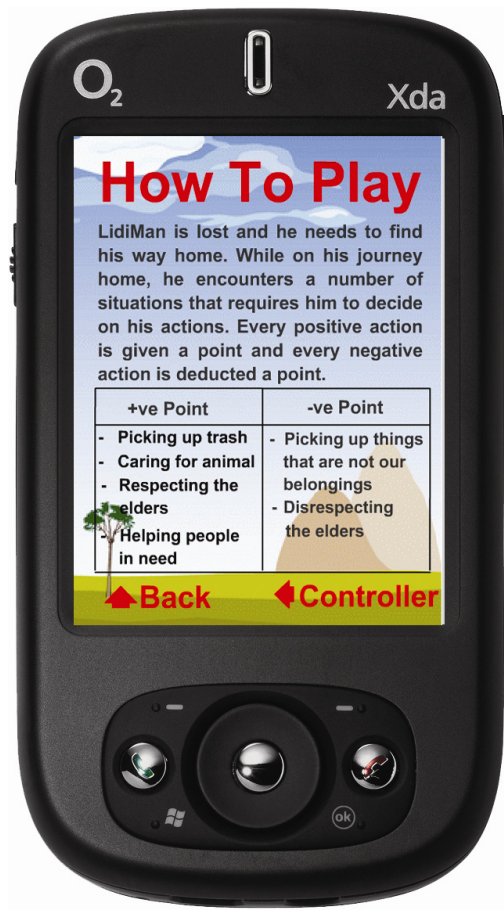

Figure 9 Keys to play (see online version for colours)

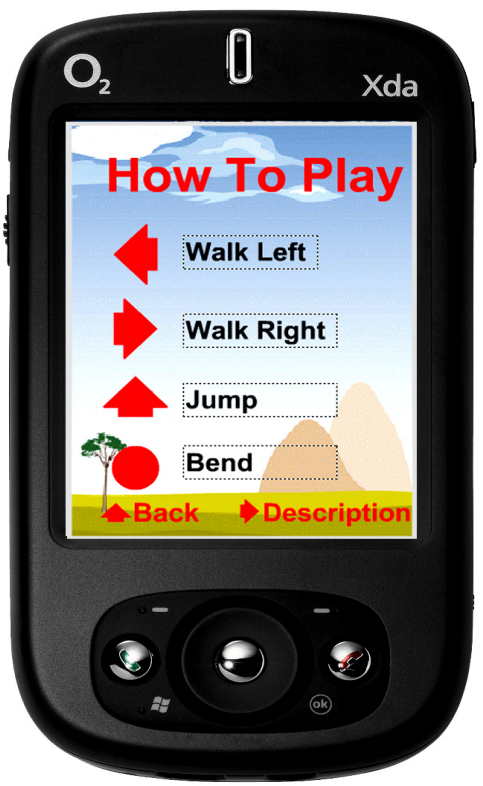


Figure 10 Respecting elderly (see online version for colours)

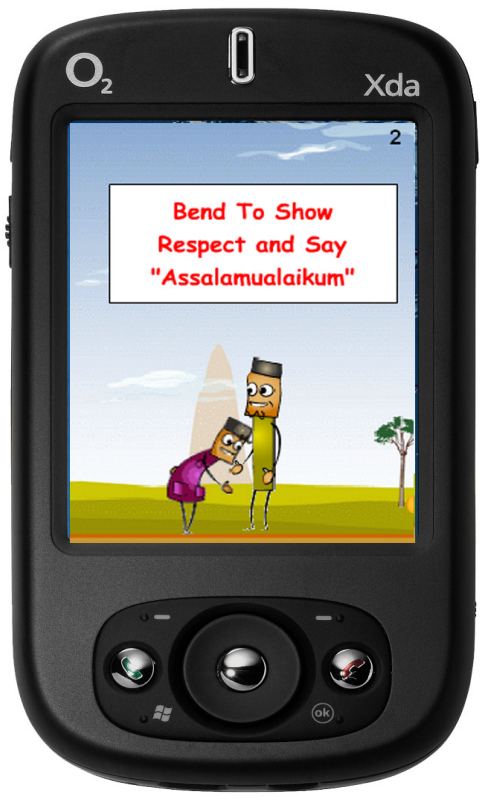

Figure 11 Helping animal gives point (see online version for colours)

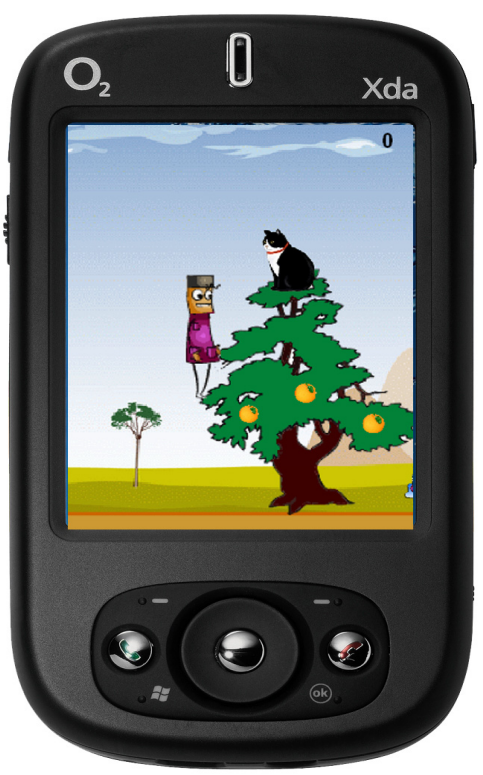


Figure 12 Picking up money will deduct point (see online version for colours)

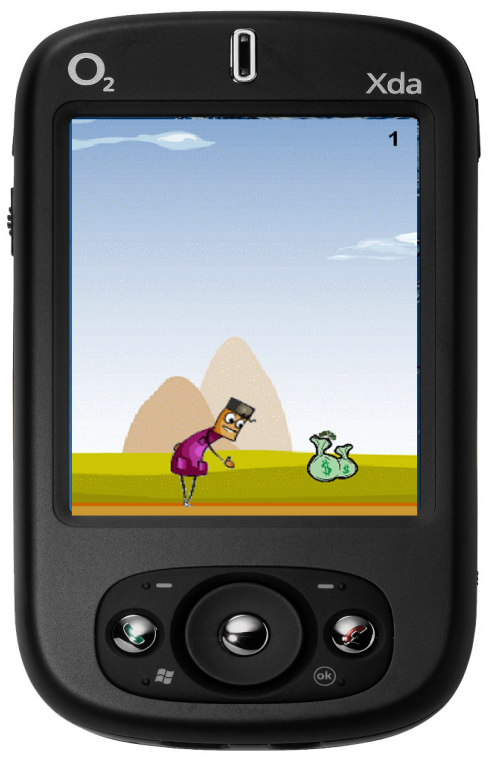

\subsection{Porting and testing MaCMoG mGame}

Among the final mGame development stages usually involve porting and testing. Porting is making changes to the game in order to make it run on different environment. In mGame development, porting is the most challenging phase where the game has to run on a large number of models and also has to cover most new models for it to be put on market. Unlike the other gaming devices, in mobile gaming there are hundreds of devices. Normally, porting can be cross platform porting (J2ME to Brew and Brew to J2ME), operator specific porting (Cingular, Vodafone, T-Mobile, Orange, Maxis, Digi, etc.) and Flash Lite and Symbian porting.

Testing a game is mandatory in ensuring there is no crash or inconvenience for players. To test a game, it is not enough to do it on emulators. Emulators cannot replace the real phones since they can only be trusted for $10 \%$ of assurance (Igda, 2005). Moreover, not all devices have emulators. Testing a game on real devices is a must for all developers to experience the screen and memory issues. It is suggested that developers test and retest the final product on real phones. Due to the nature of this fragmented medium, developers will need to access at least 10-20 mobile phones (Igda, 2005).

\subsubsection{Technical testing of $M a C M o G$}

The game testing was conducted by following the suggestions by Sloper and Burns (2002) and Rees and Fryer (2003). They categorise the testing into these dimensions:

1 Functionality: stability (Does the game crash?), correctness of game mechanics (Do the control keys function correctly?), integrity of game assets (Do multimedia elements in good quality). 
2 Compliance: formatting of standard error message, handling of memory card data, handling of legally trademarked/copyrighted materials, fulfilling particular content rating.

3 Compatibility: the game can be played on different devices?, the game can be played on different platforms? and the game can be played on different device specifications?

4 Localisation: suitability for different countries and languages.

5 Soaking: soaking testing was conducted by leaving the mGame running for a period of hours in various modes of operation, such as idling or paused without any user interaction. The purpose of soaking is to know the stability of the game.

Overall, the game technical testing was successfully conducted. As a result, the game is running properly and contains no major errors; nevertheless the mGame is only compatible with PDA and smart phone. The main objective of conducting the testing is achievable by knowing that the game is playable although on certain mobile devices.

\section{User evaluation study}

In addition to the porting and testing stage, we also conducted a user evaluation study of the mGame. Therefore, to evaluate the perception of users about MaCMoG, two main methods were used; informal interview and observation. Both evaluation methods have been conducted (at the same time) in August 2007 during the International Exposition of Research and Inventions of Institutions of Higher Learning 2007 (PECIPTA, 2007) at Kuala Lumpur. Visitors who came to our booth were randomly asked to try the $\mathrm{MaCMoG}$ and at the end of the three-day expo, 102 visitors tried the mGame. All visitors played the mGame using the devices that were provided to them.

By using informal interview and observation, generally a few key points can immediately emerge from both exercises. Immediate feedbacks are often most effective in these cases, as they allow direct actions. Thus, the main aims of the user evaluation are:

- to determine participants' perspective on $\mathrm{MaCMoG}$

- to observe participants' reactions while playing $\mathrm{MaCMoG}$

- to identify factors influencing how useful participants found the mGame.

\subsection{Informal interview}

The method of informal interviewing was used to get perspectives from participants. The interviews were conducted during this exposition because researchers were interested in seeing the participants in a natural setting while playing the mGame and to get a better sense of them without having any formal circumstances.

The questions were asked spontaneously which were basically aimed at knowing their interests in the game, game content and game characteristics (Table 3). Although, these included spontaneous questions to allow for unanticipated reactions, most of the questions were the same. Moreover, this method has also gauged the variety in participants' views. 
Table 3 Example of informal interview questions

\begin{tabular}{ll}
\hline No. & Questions \\
\hline 1 & Is the game interesting? \\
2 & Did you learn anything from the game? \\
3 & Is the game easy to play? \\
4 & Would you like to play similar game to learn in future? \\
5 & Would you buy similar game? \\
\hline
\end{tabular}

\subsection{Observation}

This method was applied to this study in order to see the participants' initial reactions and their behaviours towards $\mathrm{MaCMoG}$ and also while playing MaCMoG.

\subsection{Discussions on user evaluation}

Although, 102 participated in this stage, only 88 of them were in the target age group of this mGame. The rest were either parents accompanying their children or children aged from 5 to 8 years old, who also wanted to have a go at the mGame. Therefore, we present here the findings of only the 88 children. Figures 13 and 14 show the user evaluation study taking place at the expo. Table 4 shows the demographics of the children who participated in the study.

Figure $13 \mathrm{MaCMoG}$ poster used during evaluation (see online version for colours)

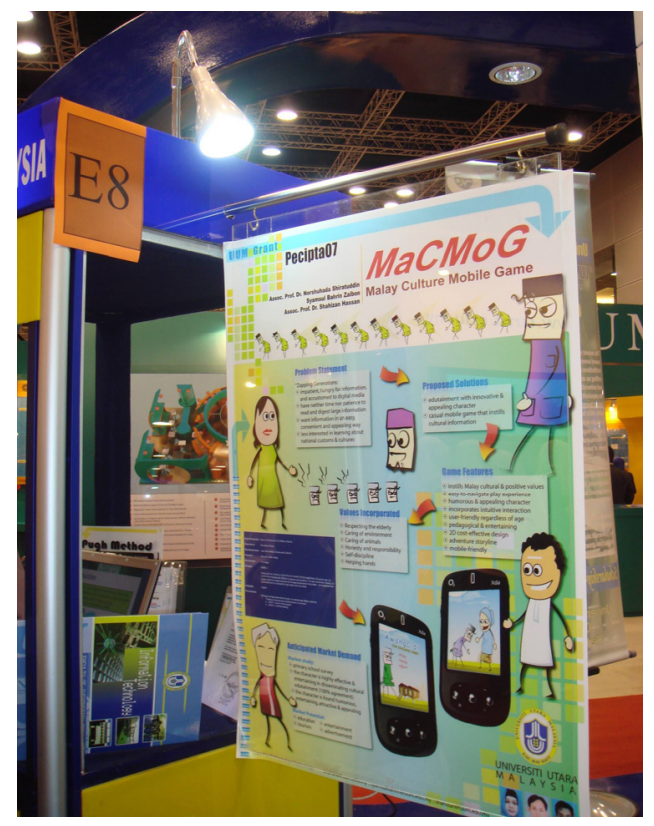


Figure 14 Participants playing MaCMoG (see online version for colours)

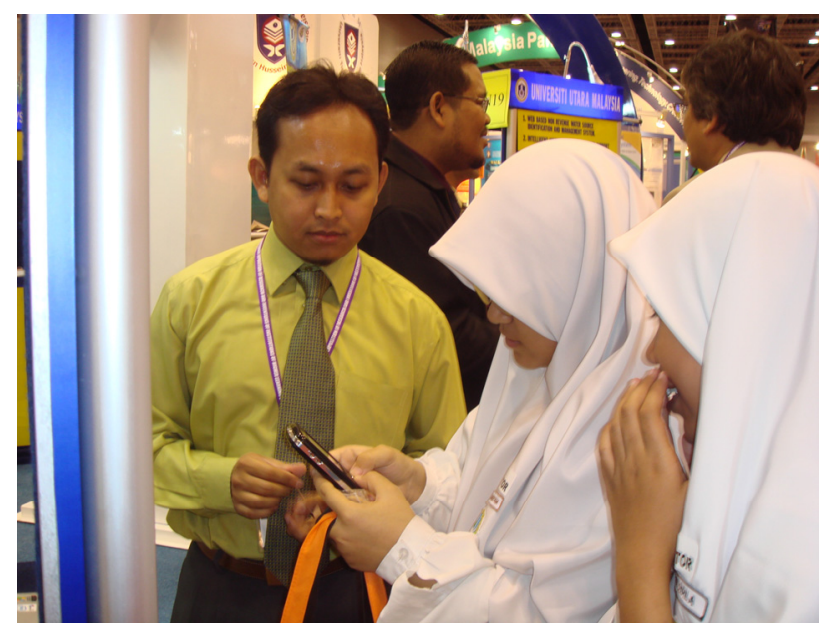

Table 4 Demographics of school students

\begin{tabular}{lccccc}
\hline Age group & No. of students & Gender & No. of students & Race & No. of students \\
\hline 9 & 21 & Male & 42 & Malay & 59 \\
10 & 22 & & & Chinese & 21 \\
11 & 23 & Female & 46 & Indian & 8 \\
12 & 22 & & & Others & - \\
Total & 88 & Total & 88 & Total & 88 \\
\hline
\end{tabular}

Based on the first and second aims of this user evaluation exercise, overall from all the participants, responses were remarkably consistent. Participants overwhelmingly considered the mGame as (refer to Figure 15):

- $\quad$ interesting and really attracted them to use or play with it

- $\quad$ successful in delivering the intended educational content

- $\quad$ easy to play and simple to use.

The majority also said they would play and buy similar game content in the future. In addition, while the evaluations were carried out, the participants' reactions were observed when they were playing the game. The average MacMoG playing time was $8.24 \mathrm{~min}$, whereby the girls needed on average an extra $2.3 \mathrm{~min}$ to get familiarised with the game concept. In general, the results of the observations indicate high interest of positive reactions to the MaCMoG. 
Figure 15 Summary of findings of the interviews (see online version for colours)

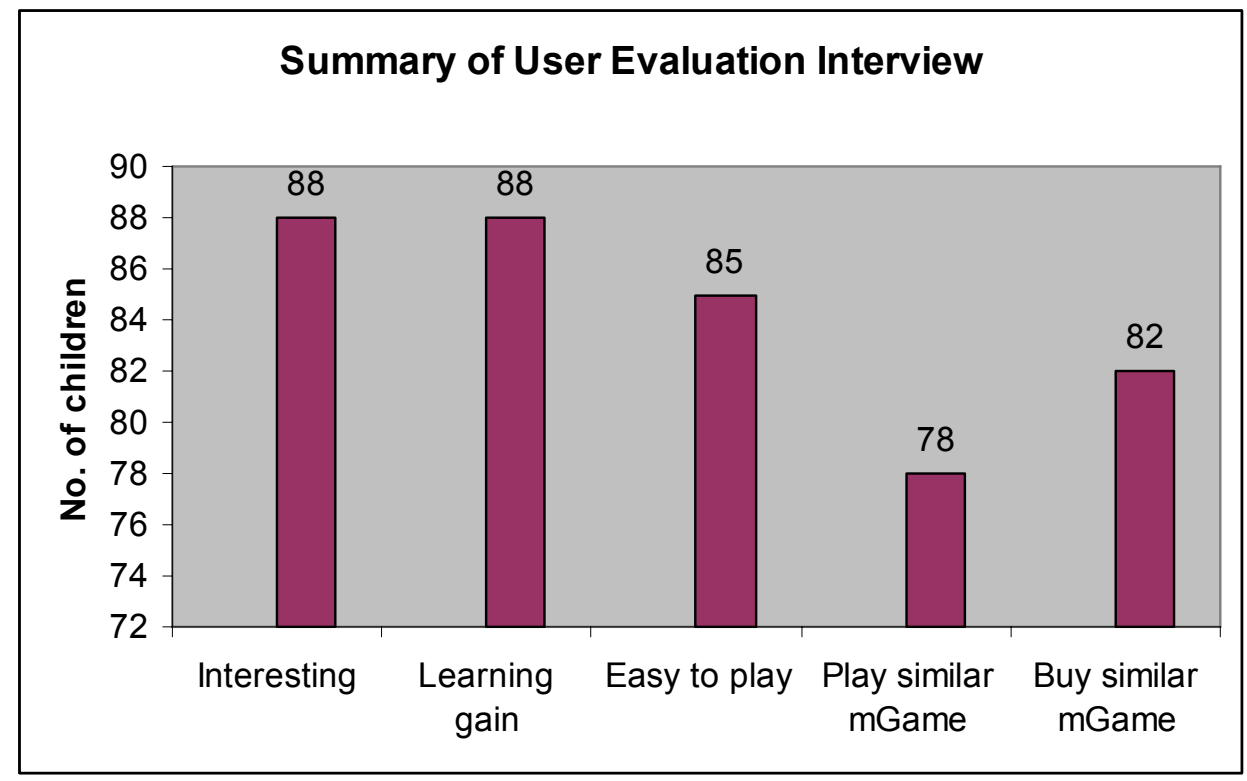

For the third objective of the evaluation, several factors were discovered to influence how useful participants found the mGame is. The factors are:

- the content is simple and easy to understand

- the content is interestingly about Malaysian environment and culture

- the local characters are appealing and well-developed, hence they are important elements to ensure better learning experience for children, youth as well as adults

- the content is perceived as matching the general concept of the proposed mGBL

- the device allowed it to run and play smoothly

- the game is unique (similar to none), so local uniqueness must always be incorporated to ensure better learning gains and increase mGBL acceptance.

\section{Conclusions}

The main deliverable of this study is the mGame itself. Although it is a prototype, the research has shown that the mGame has potential to be a learning tool and marketed since it is seen as unique to Malaysia. The characters, which have been copyrighted, are also major deliverables; they can be reused and reutilised on other games, creative applications or even TV animated series.

The instrument used for measuring the characters appealing merits can also be considered as another valuable deliverable. Testing with children often required a creative instrument in order to gauge their interests and to make explanation on 'scale' concept easier. 
MaCMoG was technically tested for these dimensions: functionality, compliance, compatibility and soaking. Furthermore, in order to assess the insights of potential players, informal interviews and observations were conducted. In addition to that, the local game characters and the game play developed for the mGame were tested for their appealing merit. Overall, responses were remarkably consistent. Participants overwhelmingly considered $\mathrm{MaCMoG}$ as interesting and really attractive, successful in delivering the intended learning content, easy to play and simple to use. The majority also said that they would play and buy similar game content in the future.

Furthermore, two important points to stress here are that

1 local, appealing and well-developed characters are important elements to ensure better learning experience for children, youth as well as adults

2 local uniqueness must always be incorporated to ensure better learning gains and increase mGBL acceptance.

A number of future considerations can be suggested for this project, for example, the focus of the game in this study is on casual game, hence, 2D characters are proposed by most experts. Exploring the same game play and storyline on 3D characters, although rather hard (Koivisto, 2007), should be contemplated to take advantage of the latest technology. Also, MaCMoG is a Flash game. Although, Flash Lite is currently available on many phone platforms such as the Symbian OS, BREW, UIQ, Windows Mobile and the Palm OS, building MaCMoG using other platforms, especially J2ME would open up a bigger market size for the mGame. Hence, a potential future project is to port MaCMoG to this platform. However, the process of porting in J2ME is different from the porting for Flash Lite. In J2ME games, developers often hard code every value for moving a sprite, for setting up background and even for setting up fonts. Moreover, since the graphics in J2ME games are bitmaps, the graphics will need to be redesigned and enhanced for a higher resolution screen size (Dholkawala, 2007).

Finally, the user evaluation study should be extended to include further data analysis such as hypothesis testing and correlation analysis. Such findings can perhaps provide richer information and more discussions.

\section{Acknowledgements}

The authors are thankful to the grant-in-aid from Universiti Utara Malaysia for providing support for this study and Shahizan Hassan for some of his ideas.

\section{References}

Abolins, M. (2006) 'Mobile games platforms explained', Pocket Gamer, Retrieved on 18 June 2007. Available at: http://www.pocketgamer.co.uk/r/Mobile/feature.asp?c=1266.

Agustin, M., Chuang, G., Delgado, A., Ortega, A., Seaver, J. and Buchanan, J.W. (2007) 'Game sketching', Conference of DIMEA '07, Perth: ACM.

Albernathy, D.J. (2001) 'Get ready for m-learning', Training and Development, Vol. 55, No. 2, pp.20-22.

Amory, A. and Seagram, R. (2003) 'Educational game models: conceptualization and evaluation', South African Journal of Higher Education, Vol. 17, No. 2, pp.206-217. 
Antonellis, I., Bouras, C. and Poulopoulos, V. (2005) 'Game based learning for mobile users', Conference of CGAIMS 2005. Louisville: Kentucky. Retrieved on 18 June 2007. Available at: http://www.stanford.edu/ antonell/papers/Antonellis_CGAIMS2005.pdf.

Ardito, C., Buono, P., Costabile, M.F., R. Lanzilotti, R. and T. Pederson, T. (2007) 'Mobile games to foster the learning of history at archaeological sites', Conference of the IEEE Symposium on Visual Languages and Human-Centric Computing (VL/HCC 2007), pp.81-86.

Attewell, J. (2005) Mobile Technologies and Learning. Shaftesbury: Technology Enhanced Learning Research Centre; Blackmore Ltd.

Bell, M., Chalmers, M., Rowland, D., Benford, S., Capra, M. and Hampshire, A. (2006) 'Interweaving mobile games with everyday life', Conference of CHI 2006, pp.417-424.

Bellotti, F., Berta, R., De Gloria, A., Ferretti, E. and Margarone, M. (2003) 'VeGamen exploring art and history in Venice', Computer, Vol. 36, No. 9, pp.48-55.

Bogost, I. (2006) 'Mobile game teaches emergency first aid', Retrieved on 18 June 2007. Available at: http://www.watercoolergames.org/archives/000579.shtml.

Budde, P. (2005) '2005 telecoms, mobile and broadband in Asia report East Timor, Indonesia, Malaysia, and Philippines', Communication Pty Ltd, KL.

Budde, P. (2007) 2007 Asia - Telecoms, Mobile and Broadband in Malaysia and Philippines, Market Research.com, Retrieved on 18 October 2007. Available at: $\mathrm{http}: / /$ www.marketresearch.com/product/display.asp?productid=1552731.

Chauhan, M. (2007) 'Developing Java-based mobile games', Retrieved on 18 October 2007. Available at: http://www.developer.com/java/j2me/article.php/3502741.

Chen, J. and Kinshuk, J. (2005) 'Mobile technology in educational services', Journal of Educational Multimedia and Hypermedia, Vol 14, No. 1, pp.91-109.

Cogoi, C., Sangiorgi, D. and Shahin, K. (2006) 'mGBL - mobile game-based learning: perspectives and usage in learning and career guidance topics', eLearning Papers, Vol. 1, No. 1, pp.1-6.

Desurvire, H., Caplan, M. and Toth, J. (2004) 'Using heuristics to improve the playability of games', CHI Conference 2004, Vienna Austria, Retrieved on 18 June 2007. Available at: http://www.behavioristics.com/downloads/usingheuristics.pdf.

Dholkawala, M. (2005) 'Cryptic capers: best practices for mobile game development', Retrieved in 18 June 2007. Available at: http://www.adobe.com/devnet/devices/articles/cryptic capers.html.

Dholkawala, M. (2007) 'Porting J2ME games to Flash Lite', Adobe Developer Connection. Retrieved on 18 July 2007. Available at: http://www.adobe.com/devnet/devices/articles/ porting_j2me_flashlite.html.

Di Blas, N., Paolini, P. and Poggi, C. (2005) '3D worlds for edutainment: educational, relational and organizational principles', Third IEEE International Conference Pervasive Computing and Communications Workshops, pp.291-295.

Fabricatore, C. (2000) 'Learning and videogames: an unexploited synergy', The International Conference of the Association for Educational Communications and Technology, Denver.

Forum Nokia (2004) 'Usability in mobile games', Game User Experience Library, Retrieved on 18 June 2007. Available at: http://www.forum.nokia.com/main/html_readers/usability_and_ fun.html.

Igda (2005) 'Mobile game white paper', International Game Developers Association, Game Developers Conference. Retrieved on 18 June 2007. Available at: http://www.igda.org/online/ IGDA_Mobile_Whitepaper_2005.pdf.

Janousek, S. (2007) 'The art and zen of mobile game creation with Flash', Slideshare.net.

Jo, T. (2007) 'MDeC boosting creative content growth', The Star, Tuesday, 28 August 2007.

Kam, M., Ramachandran, D., Devanathan, V., Tewari, A. and Canny, J. (2007) 'Localized iterative design for language learning in underdeveloped regions: the PACE framework', Conference of CHI 2007, NY, USA: ACM Press. 
Kneunster, D. (2004) 'Storyboarding notes: a bunch of short guys', Retrieved on 19 July 2007. Available at: http://www.abunchofshortguys.com/.

Koivisto, E. (2007) 'Mobile Game 2010', Nokia Research Centre. Retrieved on 19 July 2007. Available at: http://research.nokia.com/files/NRC-TR-2007-011.pdf.

Koller, A. (2003) Java ME vs. Flash Lite: A Comparison of Mobile Phone Game Development. South Africa: Rhodes University.

Korhonen, H. and Koivisto, E. M. I (2007) 'Playability heuristics for mobile multi-player games', Conference of the 2nd International Conference on Digital Interactive Media in Entertainment and Arts, ACM International Conference Proceeding Series, Vol. 274, Perth, Australia, pp.28-35.

Lee, J-H. (2005) 'The educational use of mobile games', Conference of OLT 2005 Conference, Queensland University of Technology, Brisbane, pp.143-149.

Maniar, N. and Bennett, E. (2007) 'Designing a mobile game to reduce 'culture shock', Advances in Computer Entertainment Technology, Advances in Computer Entertainment Technology, Volume 203 of ACM International Conference Proceeding Series. pp.252-253.

Massie, A. (2005) Mobile Phones - Education, Creative Industries Faculty, QUT.

Md Saidin, A.I. and Amira, S.F. (2006) Importance of Capacity Building for the Digital Content Industry in Malaysia. Kuala Lumpur: University Malaya.

Mencher, M. (2004) The Future of Game Development: New Skills And New Attitudes Part 1: Mobile Games, GIGnews.com.

Norshuhada, S., Syamsul Bahrin, Z. and Shahizan, H. (2007) 'Malay culture mobile game', Exposition of PECIPTA, Kuala Lumpur.

Novak, J. (2004) Game Development Essentials: An Introduction. New York: Thomson Delmar Learning.

Pelkonen, T. (2004) 'Mobile games'. E-Content Report 3, ACTeN. Retrieved on 18 June 2007. Available at: http://www.acten.net/uploads/images/382/IR3_010304-2.pdf.

Prensky, M. (2001) Digital Game-Based Learning. New York: McGraw-Hill.

Ransley, W. (1991) 'An instrument for measuring five aspects of children's attitudes towards microcomputers', British Journal of Educational Technology, Vol. 22, No. 3, pp.216-221.

Raybourn, E. and Wærn, A. (2004) 'Social learning through gaming', Extensive Abstracts CHI 04, pp.1733-1734.

RealNetworks $^{\circledR}$, Inc. (2006) 'Research reveals casual games provide mental balance, stress relief and relaxation', RealNetworks, Retrieved on 18 June 2007. Available at: http://www.realnetworks.com/company/press/releases/2006/casgames_research.html.

Rees, E. and Fryer, L. (2003) 'Best practices in quality assurance/testing', Retrieved on 10 November 2007. Available at: http://www.igda.org/qa/docs/IGDA_Best_Practices_QA.pdf.

Roslan, M. (2007) Incentives for Malaysian Content Development, Putrajaya: Content and Industry Development MyICMS, Technology and Standard Division, Suruhanjaya Komunikasi Multimedia Malaysia.

Sánchez, J., Salinas, A. and Sáenz, M. (2007) 'Mobile game-based methodology for science learning', Lecture Notes in Computer Science, Vol. 4553, pp.322-331.

Schwabe, G. and Goth, C. (2005) 'Mobile learning with a mobile game: design and motivational effects', Journal of Computer Assisted Learning, Vol. 21, pp.204-216.

Semenov, A. (2005) 'Mobile games', Research Seminar on Telecommunications Business II, Helsinki University of Technology, Retrieved on 18 June 2007. Available at: http://www.tml.tkk.fi/Opinnot/T-109.551/2005/reports/Mobile_Games.doc.

Sloper, T. and Burns, M.S. (2002) 'Sloperama's game biz advice page', Retrieved on 10 November 2007. Available at: http://www.sloperama.com/advice/index.html.

Ward, A. (2005) Game Character Development with Maya. Berkeley: New Riders.

W2Forum.com (2005) Mobile Content. Wireless World Forum. 
Yuan, M.J. (2003) Develop State-of-the-Art Mobile Games, JavaWorld.com. Retrieved on 10 March 2007. Available at: http://www.javaworld.com/javaworld/jw-11-2003/jw-1107wireless.html?page $=1$.

Zhang, W., Han, D., Kunz, T. And Hansen, K.L. (2007) 'Mobile game development: objectorientation or not', Conference of Computer Software and Applications Conference, COMPSAC 2007, Vol. 1, pp.601-608.

\section{Bibliography}

Parda, A. (2006) 'Wanted: killer application for 3G', Computimes, NST, 3 March. 